\title{
Evidence for an Allelopathic Interaction Between Rye and Wild Oats
}

Francisco A. Macías, ${ }^{* \dagger}$ Alberto Oliveros-Bastidas, ${ }^{\dagger \dagger}{ }^{\dagger}$ David Marín, ${ }^{\dagger}$ Nuria Chinchilla, ${ }^{\dagger}$ Diego Castellano, ${ }^{\dagger}$ and José M. G. Molinillo ${ }^{\dagger}$ †Allelopathy Group, Department of Organic Chemistry, Institute of Biomolecules (INBIO), Campus de Excelencia Internacional
Agroalimentario (ceiA3), University of Cadiz, C/Avda. República Saharaui, no. 9, 11510 Puerto Real, (Cádiz), Spain
${ }^{\ddagger}$ Grupo de Química Ecológica, Departamento de Química, Universidad de Los Andes, Núcleo Universitario Pedro Rincón Gutiérrez,
La Hechicera, 5101-A Mérida, Venezuela

Supporting Information

ABSTRACT: Allelopathy is a biological phenomenon in which an organism produces one or more biochemicals that influence the growth, survival, and reproduction of other organisms. Allelopathy has been the subject of a great deal of research in chemical ecology since the 1930s. The characterization of the factors that influence this phenomenon has barely been explored, mainly due to the complexity of this area. The main aim of the research carried out to date has been to shed light on the importance of these interactions in agroecosystems, especially in relation to the interactions between crops and weeds. Herein we report the characterization of a complete allelochemical pathway involving benzoxazinones, which are known to participate in allelopathic plant defense interactions of several plants of high agronomic interest. The production of the defense chemicals by a donor plant (crop), the route and transformations of the chemicals released into the environment, and the uptake and phytotoxic effects on a target plant (weed) were all monitored. The results of this study, which is the first of its kind, allowed a complete dynamic characterization of the allelopathic phenomenon for benzoxazinones.

KEYWORDS: allelopathy, interactions, phytotoxic, benzoxazinones, Gramineae

\section{INTRODUCTION}

Allelopathy is the science that concerns the study of any process involving secondary metabolites produced by plants, algae, bacteria, and fungi that influence the growth and development of agricultural and biological systems. Allelopathy encompasses a wide range of disciplines related to plant studies, including ecology, biochemistry, chemistry, plant physiology, technical agriculture, forestry, and soil studies. ${ }^{1}$ The ecological consequences of the allelopathic phenomenon are diverse and they include plant predominance, exotic invasion, and forest succession. ${ }^{2}$ Root exudation of small molecules plays a major role in plant ecosystems and it is often associated with the development of competitive advantage through allelopathy. ${ }^{3}$ However, allelopathy seems to be more important in exotic invasion than in natural communities, so it is possible that interactions between plant species may drive natural selection in communities, ${ }^{4}$ with this coevolution playing a role in the manifestation of allelopathy. ${ }^{5}$

Another area in which allelopathy plays a major role is in the interaction of crops and weeds. A common characteristic of plant allelochemicals is phytotoxicity, and the release by crop plants with subsequent uptake by weeds or invasive plants is likely to be relevant in crop production. Allelochemicals have been proposed and used as templates for the development of novel phytotoxic molecules with potential agronomic utility.

The identification of compounds involved in allelopathic interactions requires complex experimentation, especially when the chemicals of interest are exuded below ground. The complexity of the allelopathic phenomenon is still far from being fully understood. The ideal situation, in which one plant exudes an allelochemical, the receptor absorbs it, and the substance provokes an effect on the target plant without any other influence, cannot be found in nature. ${ }^{6}$ Allelochemicals can reach the soil through leaching from aerial parts, decomposition of donor plant residues, or by means of root exudation, with the latter mechanism recently receiving increased attention. As it is closely related to root exudation, the role of soil microorganisms has also attracted increased interest. Soil microbes could transform the exuded compound into other compounds that may be more or less active than the compound originally released by the donor plant. ${ }^{7}$ An increase in the phytotoxic effect after biotransformation has been documented in some cases. $^{8}$

Neither field observations nor ecological studies serve to establish allelopathy if they are not substantiated by an understanding of the chemicals involved. However, allelochemicals themselves are nothing but phytotoxic compounds if they are taken out of their context and their release and uptake by target plants are not demonstrated.

The main objective of the study described here was to assess the allelochemical pathway of benzoxazinones. This investigation involved measuring the concentration of each allelochemical within the donor plant, their exudation dynamics, their concentrations in soil (including degradation products) at different times during the process, their absorption by a target organism, and the evaluation of the phytotoxicity caused by the chemicals first released by the donor plant and/

Received: May 29, 2014

Revised: September 8, 2014

Accepted: September 9, 2014

Published: September 9, 2014 


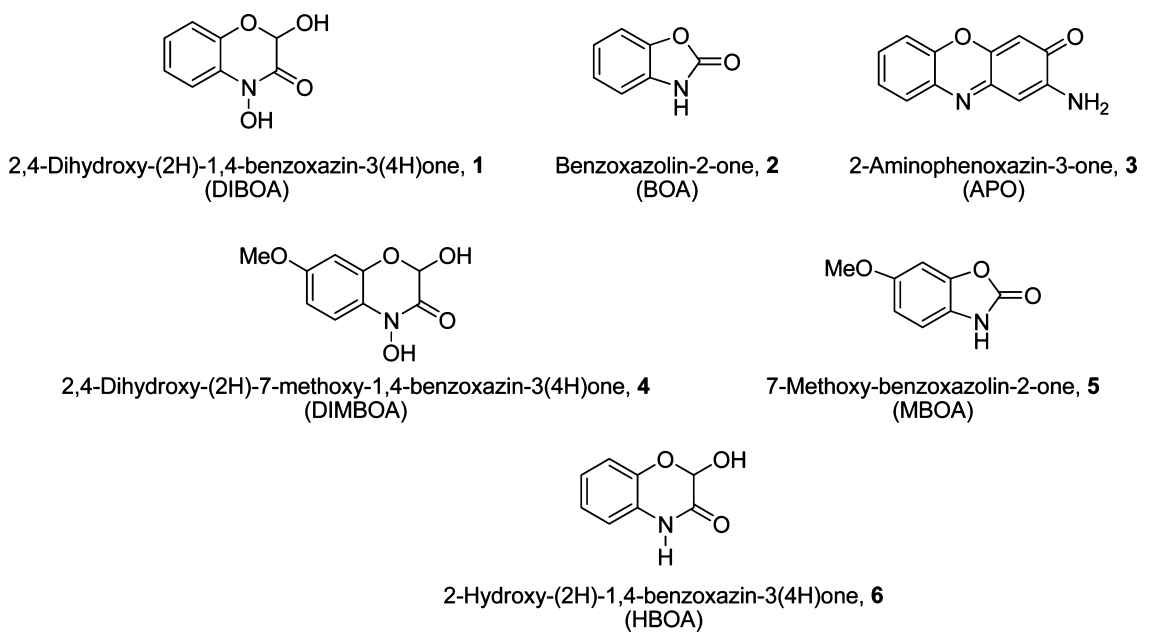

Figure 1. Structures of the compounds under discussion.

or their degradation products, as characterized inside the target plant. All of the analyses were conducted in a single bioassay involving a coculture of both species in order to ensure direct and mutual interactions between the donor and target plant. The dynamic character of the different determinations performed adds the value of a real-time description of the various processes that are involved in the allelopathic phenomenon.

The study focused on the interaction of rye (Secale cereale L.), as a strategic crop, with one of its most common weeds (wild oat, Avena fatua L.). This interaction is mediated by benzoxazinone allelochemicals, and we propose a relevant role for the degradation products on the basis of the experimental results. ${ }^{9}$ S. cereale is well-known as a producer of 2,4-dihydroxy$(2 H)^{-1}$,4-benzoxazin-3(4H) one (DIBOA, 1) (Figure 1). Extensive research has been performed on the phytotoxic effects of this compound and its degradation products benzoxazolin-2-one (BOA, 2) and 2-aminophenoxazin-3-one $(\mathrm{APO}, 3){ }^{10,11}$ as well as the fate of allelochemicals ${ }^{12,13}$ and the physiological aspects of the mode of action. ${ }^{14-16}(2 \mathrm{H})-1,4-$ Benzoxazin-3(4H)-ones have been isolated from Poaceae, Acanthaceae, Ranunculaceae and Scrophulariceae plants. Indeed, degradation in different systems, such as aqueous solutions, ${ }^{7,11,17}$ microbial biotransformation, ${ }^{18}$ or crop soils, ${ }^{7,11,19,20}$ has been widely studied along with the dynamics of these systems. In addition, these compounds have shown a wide spectrum of biological activities, ${ }^{21-24}$ as have some of their degradation products ${ }^{25,26}$ and a wide variety of synthetic analogs. ${ }^{27}$ Compounds such as 2,4-dihydroxy-(2H)-1,4-benzoxazin-3(4H)-one (DIBOA, 1) and its 7-methoxy analog (DIMBOA, 4) have been described as chemicals with relevant roles in chemical ecology for the last 50 years. ${ }^{9}$ The levels of DIBOA (1), BOA (2), and APO (3) were monitored in $S$. cereale, culture medium, and $A$. fatua, and the results were correlated with the phytotoxic effects observed.

\section{MATERIALS AND METHODS}

Statistical Analysis. Data for the lengths of root and shoot were statistically analyzed using Welch's test, with significance fixed at 0.01 and 0.05 . The results are represented in bar charts as percentages from the control. Thus, zero represents the control, positive values represent stimulation of the studied parameter, and negative values represent inhibition. The fresh weights of seedlings were determined in triplicate, and the mean and standard deviation were calculated. A similar procedure was followed for endogenous (root and shoot content) and exogenous (exudate content) contents of the different metabolites. The quantitation of DIBOA (1), BOA (2), and APO (3) was carried out in quintuplicate using calibration curves and outlying values were discarded (values out of the range of the spiked value $\pm 20 \%)$.

Bioassays. S. cereale-A. fatua Pregermination. The seeds used for the coculture experiment were surface-sterilized by soaking for 30 min in calcium hypochlorite $75 \mathrm{~g} / \mathrm{L}$ (Merck, Darmstadt, Germany) with traces of Tween 20 (Sigma-Aldrich, St. Louis, MO, U.S.A.) followed by rinsing with sterilized deionized water. The sterilized seeds were placed in Petri dishes ( $8.5 \mathrm{~mm}$ diameter) containing a semisolid culture medium from Sigma-Aldrich (Agar-agar 7 g/L, Hoagland No. 2 basal salt mixture, $\mathrm{pH}$ 5.7). Petri dishes were placed in a growth chamber Celsius 2000 (Memmert, Duesseldorf, Germany) for a $16 \mathrm{~h}$ photoperiod at $25{ }^{\circ} \mathrm{C}, 70 \%$ relative humidity, with cold white light (standard illuminant D65; $6500 \mathrm{~K}$ ). The activities were assessed under sterile conditions.

S. cereale-A. fatua Coculture. The bioassay substrate was designed by considering two criteria: an accurate simulation of the field interaction conditions and ease of monitoring the exuded chemicals. Glass perlite $(3 \mathrm{~mm}$ diameter, $200 \mathrm{~g}$ ) was placed in a glass flask $(2.5 \mathrm{~L})$ and culture solution (Hoagland No. 2 basal salt mixture, $\mathrm{pH} 5.7,300$ $\mathrm{mL}$ ) was added. Glass perlite was chosen due to its negligible interference on the analysis by adsorption or absorption of the test solution and also because it minimizes the sample conditioning operations required prior to analysis. In this respect, a simple filtration was sufficient, and this avoided the need for extraction processes with organic solvents. Minimization of the absorption or adsorption of chemicals also enhances their bioavailability. Seedlings of A. fatua ( $3 \mathrm{~d}$ old) and $S$. cereale ( $6 \mathrm{~d}$ old) were placed inside the flasks, and they were allowed to grow for $6 \mathrm{~d}$. The number of A. fatua seedlings was fixed at 4 and the number of $S$. cereale seedlings was set up according to the following donor/target plant ratios: 1:1, 2:1, 3:1, 7:1, 10:1, and 20:1. These donor/target proportions were optimized for maximum effect by performing bioassays under identical culture conditions. A. fatua and $S$. cereale monocultures, prepared under analogous conditions, were used as controls for phytotoxicity and DIBOA (1) production measurements.

Phytotoxicity Parameters. Phytotoxicity on A. fatua was measured by comparing its root length, shoot length, and shoot fresh weight after exposure to $S$. cereale seedlings under the experimental conditions described above. In addition, potential effects of $A$. fatua on $S$. cereale were studied and described in terms of root length, shoot fresh weight, and DIBOA (1) content.

Analytical Method. DIBOA (1), BOA (2), and APO (3) levels in culture solution were recorded using the previously described HPLC$\mathrm{DAD}$ method. ${ }^{28}$ Quantitation was performed using a method with an external standard. BOA (2) was purchased from Fluka Chemika Co. 
(Sigma-Aldrich, St. Louis, MO, U.S.A.). DIBOA (1) and APO (3) standards were obtained by the previously described methodology. ${ }^{29}$ Compounds were identified by comparison of retention times and $\mathrm{UV} /$ vis spectra at two wavelengths, namely 253 and $264 \mathrm{~nm}$ (95\% confidence), using external and internal standards. A single analysis therefore provided a description of the chemical situation of the whole system at a given time, and subsequent repetition of the analysis provided a complete description of the chemical evolution in terms of production and exudation by the donor plants (S. cereale), assimilation by the target plant (A. fatua) and degradation or retention by the environment.

DIBOA Detection and Quantitation in A. fatua. These experiments were performed on a Inova 600 NMR spectrometer (Varian, Palo Alto, CA, U.S.A.) equipped with an interchangeable triple-resonance microflux probe (Varian ${ }^{1} \mathrm{H}\left[{ }^{13} \mathrm{C} /{ }^{15} \mathrm{~N}\right] 3 \mathrm{~mm}$ pulsedfield gradients PFG, $60 \mu \mathrm{L}$ active volume) and connected to a Varian HPLC-UV/MS system (Varian 1200L quadrupole mass spectrometer, Varian HPLC-UV system). The UV/vis outlet was connected to the NMR equipment by means of a Rheodyne sampling unit. Stop-flow experiments were performed by using a Valco stop-flow valve. This valve was controlled by the LC-NMR software, considering the UV/vis and NMR detector delay. Liquid chromatography was carried out using an analytical column $150 \mathrm{~mm} \times 4.6 \mathrm{~mm}$ i.d., $5 \mu \mathrm{m}$ Gemini $110 \mathrm{~A}$ RP-18 (Phenomenex, Torrance, CA, U.S.A.) with a guard column SecurityGuard Cartridges Gemini RP-18 (Phenomenex, Torrance, CA, U.S.A.) under reverse phase and isocratic conditions with a mixture of $\mathrm{MeOH}$ (1\% TFA): $\mathrm{H}_{2} \mathrm{O}$ (1\% TFA) (7:3). Gradient separation was performed using (A) $\mathrm{MeOH} ; 1 \%$ TFA and (B) $\mathrm{H}_{2} \mathrm{O} ; 1 \%$ TFA with the following elution pattern: $t_{0}=30 \% \mathrm{~B} ; t=40 \mathrm{~min}, 100 \% \mathrm{~B}$. The system was operated at room temperature and the eluent flow was set to 0.8 $\mathrm{mL} / \mathrm{min}$. ${ }^{1} \mathrm{H}$ NMR spectra were recorded under on-flow and stop-flow conditions for standard solutions and A. fatua tissue extracts, respectively. Solvent suppression was performed by using the WET2 method. Thirty-two NMR accumulations were used for standards and 256 for samples. The spectral width was set to $10000 \mathrm{~Hz}$ in all experiments. WETG-COSY spectra were acquired using 48 accumulations, 128 and $2 \mathrm{~K}$ increments with a $10000 \mathrm{~Hz}$ spectral width in both dimensions and a $1 \mathrm{~s}$ relaxation time. $\mathrm{CD}_{3} \mathrm{OD}$ was used as the solvent and the residual $\mathrm{MeOH}$ peak $(\delta 3.30 \mathrm{ppm})$ was used as a reference. A $10 \mu \mathrm{L}$ injection of an equimolecular mixture of DIMBOA (4), DIBOA (1), BOA (2), and MBOA (5) (standard solution) was carried out. A. fatua methanol extracts were used for allelochemical detection and these were injected at a concentration of $3 \mathrm{mg} / \mathrm{mL}$.

\section{RESULTS AND DISCUSSION}

Experimental Design. The overall objective of this study was to characterize completely the allelopathic pathway with living plants and real exudates. To achieve this aim, we developed an experimental design that balances two factors: to model the real phenomenon under laboratory conditions and to mimic the real situation as closely as possible. The design allowed us to record the following information: the release of allelochemicals into the culture substrate in bioactive concentrations, monitorization of the degradation phenomena, identification of the final products, detection of the uptake of the "bioactive cocktail" by the target plant, and, finally, a beneficial or detrimental effect described using appropriate parameters. The experimental design was optimized by fixing the following elements: allelochemicals, donor plant, target plant, and substrate.

Donor Plant Selection. Wheat, rye, and maize have shown allelopathic effects, and they produce benzoxazinones. Farmers have taken advantage of these properties by employing them for crop rotation and by using their residues to treat the soil. ${ }^{30}$ The activities of some mulches have also been tested. The effects of soil-incorporated wheat on the germination of 12 different weed species have been studied. ${ }^{31}$ These allelochem- icals have two desirable features for the present study: first, it is possible to measure accurately their concentrations in the donor plant, target plant, and the environment in which they grow; second, their degradation products are known and can be measured simultaneously with the initially released allelochemicals. Fortunately, a great deal of research has been focused on the bioactivity and biodegradability of these compounds and this has led to one of the most in-depth studies published to date. $^{27}$

A key point in the selection of the donor plant is the stability of the allelochemicals present. Thus, the persistence of DIBOA (1) in soil and other environments is much higher than that of DIMBOA (4). The half-life of DIBOA (1) is $62 \mathrm{~h}$ in soil, $48 \mathrm{~h}$ in water and $12 \mathrm{~h}$ in bioassay media, ${ }^{7}$ whereas the corresponding values for DIMBOA (4) are $31 \mathrm{~min}, 18$ and 8 h, respectively. ${ }^{11}$ Wheat and rye both produce DIMBOA (4) and DIBOA is also produced in the case of wheat. A higher level of activity was found in $S$. cereale and the major benzohydroxamic acid produced for this species is the more stable DIBOA (1). ${ }^{32}$ These facts led us to select $S$. cereale as the donor plant.

Target Plant Selection. It has been reported that the following plant species, among others, are sensitive to DIBOA (1): Lepidium sativum, Eruca sativa, Lactuca sativa, Sinapis alba, Vicia hirsute, Spinacia oleracea, Raphanus sativus, Brassica rapa var. oleifera, Lolium rigidum, Brassica rapa var. esculenta, Avena fatua, and Echinochloa crus-galli. Exploratory bioassays were performed in order to select the target species and Avena fatua was found to be the optimal model for several reasons: this weed has rapid growth and biomass development (which facilitates extraction and analysis) and it has been used for phytotoxicity tests under field conditions for Secale cereale and Triticum aestivum. Finally, this species interacts in the field with Gramineae crops and is classified as one of the most problematic weed species.

Influence of Seedling Density on the Experimental Design. Phytotoxicity data recorded on A. fatua for the different $S$. cereale seedling densities are shown in Figure 2.

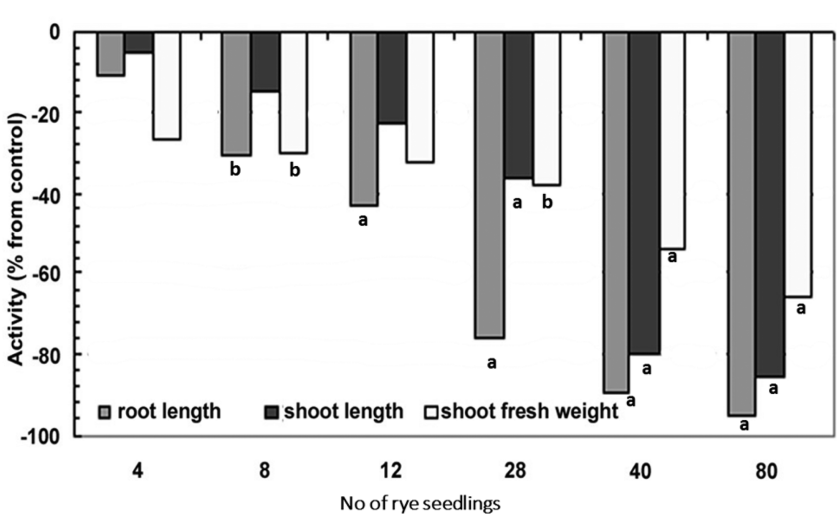

Figure 2. Phytotoxic activity on A. fatua seedlings in coculture bioassay with $S$. cereale.

Dose-response effects could be observed and mathematically treated (Figure 3). The presence of 14 S. cereale seedlings caused 50\% inhibition of root length, whereas 35 seedlings were needed to cause the same level of toxicity when measured on shoot length. The growth parameters and the DIBOA (1) levels were evaluated for the donor plant, as some interaction caused by the organism chosen as the target could also take place. In 


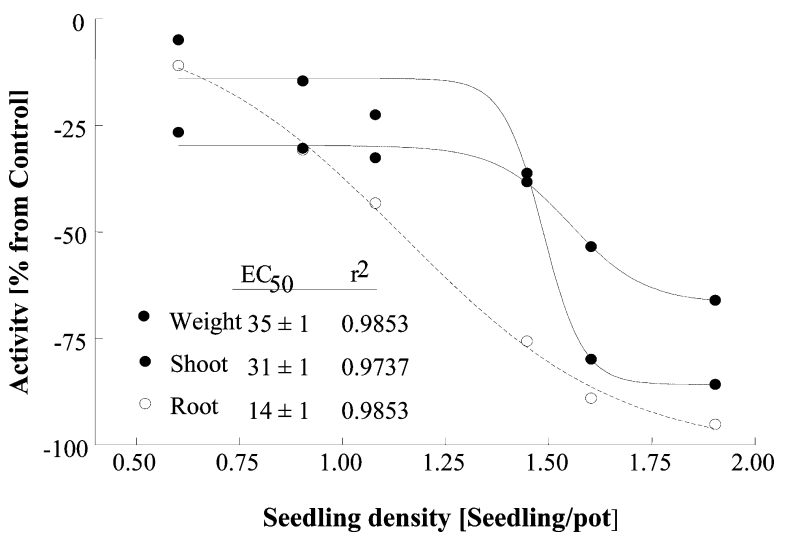

Figure 3. Dose-response curves for A. fatua growth vs S. cereale ratios.

control samples, the number of seedlings of the target species was replaced by the same number of rye seedlings.

The effects on weight, length, and the accumulation of DIBOA (1) for S. cereale in coculture with A. fatua are shown in Figure 4. In general, growth inhibition was very low, with the highest effect at around $12 \%$. As can be observed, at lower donor seedling densities the growth parameters were inhibited to a greater extent. This trend is consistent with a detrimental effect caused on the donor plant. In addition, endogenous DIBOA (1) production decreased when the proportion of the target plant was decreased. This result is consistent with production of the defense metabolite being triggered by the presence of the target organism. The mutual character of the allelopathic interaction was therefore clearly displayed, and the plants chosen as donor and target played their expected roles under the selected experimental conditions.

The study of the chemical interference between two species continued with the detection of the allelochemicals in the donor plant exudates. Chromatograms were obtained for exudates at three ratios of donating seedlings in the coculture and the amounts of DIBOA (1), BOA (2), and APO (3) were also determined. In general, the levels of DIBOA (1) are similar to those obtained without target species although they do tend to be slightly lower in the latter case. A progressive increase in the amounts of DIBOA (1) in the media was observed on increasing the density of donating seedlings. This trend was less marked in the cases of BOA (2) and APO (3). In terms of concentration units, defined by the volume of initial nutrient solution, the highest concentration of DIBOA (1) in the system was $0.3 \mathrm{mM}$. Under these experimental conditions, the exudation, absorption, changes in solution volume, and degradation generated a phytotoxic concentration gradient that affected the target organism rather than a fixed concentration. These conditions are much more similar to an environmental allelopathic interaction than those used in most dose-response bioassays, in which there is a fixed concentration of a potentially active substance.

Once we had dynamically determined the donor plant exudation potential, degradation phenomena led to changes in the composition of the culture solution. In addition to the chemicals identified, DIMBOA (4) and the lactam HBOA (6) were detected (Figure 5). The presence of DIMBOA (4) in

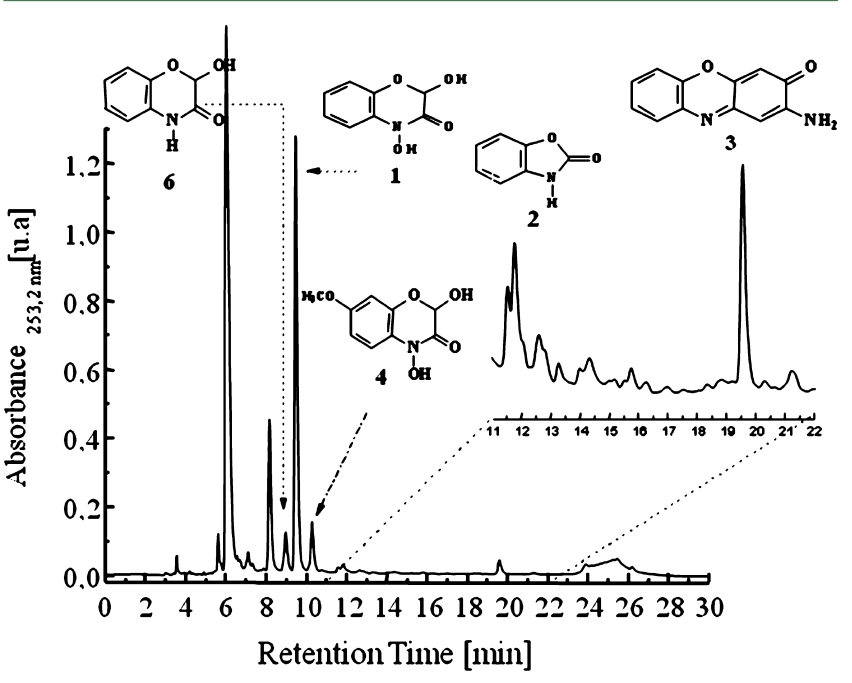

Figure 5. Chromatographic profile of the growth medium in coculture of $S$. cereale and A. fatua. Conditions: 300 seedlings, $12 \mathrm{~d}$ of growth from germination.

roots of rye and its exudates has been discussed. The production of this compound by seedlings seems to be dependent upon genetic and environmental factors as well as the bioassay design. In addition, secondary metabolites, and particularly the production of benzoxazinones in crops, have been characterized as highly accession-dependent, as indicated

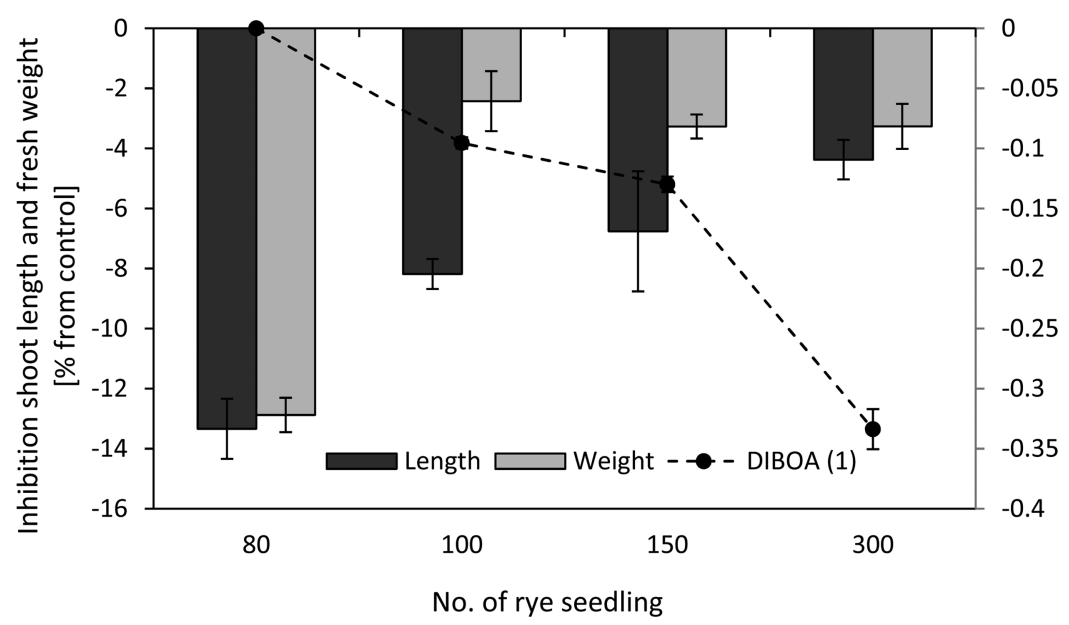

Figure 4. Effects on growth and accumulation of DIBOA (1) in the S. cereale bioassay. 


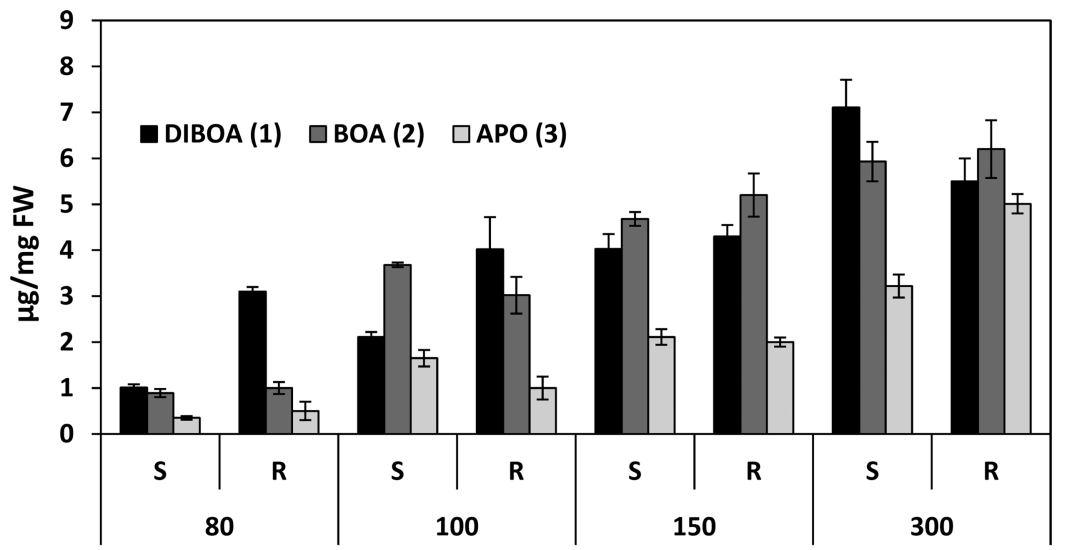

No. of seedling of S. secale [Seedlings/pot]

Figure 6. Levels of DIBOA (1), BOA (2), and APO (3) in shoot and root of A. fatua for different densities of S. cereale.

by a particular study on benzoxazinones from wheat. ${ }^{33}$ Thus, in this case the phytotoxic effects on the target plant must correspond partly to the action of DIBOA (1), DIMBOA (4), and HBOA (6). Nevertheless, qualitatively it is evident that the DIBOA (1) concentration in the exudates was much higher than those of DIMBOA (4) and HBOA (6). Moreover, the phytotoxicity of DIBOA (1) to A. fatua was found to be much higher than that found for the other two benzoxazinones. ${ }^{34}$ Therefore, DIBOA (1) and related compounds should be the most relevant molecules in considering phytotoxic effects and, consequently, the analysis was focused on these compounds.

DIBOA (1) and its degradation products BOA (2) and APO (3), present in the culture medium were studied inside the target plant tissue. The results of this study provided information on the contribution of each chemical to the observed phytotoxicity and confirmed their previously suspected $^{35}$ contribution to the interaction phenomena mediated by chemicals.

Time Dependence. As mentioned above, the concentrations of allelochemicals in the culture medium depended on the exudation rate, changes in the solution volume during the bioassay, and degradation of the primary products released by the donor plant. Thus, the degradation processes affected the amount of allelochemical by diminishing its concentration, whereas the consumption of nutritient solution caused an increase in the concentration in the system. These findings are not unique to this particular bioassay. In nature, it has been estimated that the concentrations of compounds are also in a gradient, with maximum and minimum values defined by the dynamics of the exudation phenomenon and the transformation processes. ${ }^{36,37}$ This phenomenon was also studied in some detail by considering culture solution consumption for a density of 50 seedlings during $12 \mathrm{~d}$ of growth. It was found that a volume of $10 \mathrm{~mL}$ was sufficient for regular plant development. In the present bioassay, $60 \mathrm{~mL}$ of culture solution was used. This approach allowed us to infer that $10 \mathrm{~mL}$ of solution must still remain at the end of the bioassay with 300 seedlings (12 d).

Unambiguous Identification. The identification criteria in the analysis of DIBOA (1) in the nutrient solution were based on the commonly used chromatographic methodologies: retention time, addition of standard to the sample, recording of the ultraviolet spectrum, and comparison with pure standards. The unambiguous determination of the compound in target plant extracts was ensured by combining liquid chromatography with UV/vis spectrophotometry, mass spectrometry, and nuclear magnetic resonance spectroscopy. A full analytical system based on these techniques was designed and this provided the UV/vis spectrum, mass spectrum, and ${ }^{1} \mathrm{H}$ NMR and ${ }^{1} \mathrm{H}-{ }^{1} \mathrm{H}$ 2D WET-gCOSY NMR spectra. All of the techniques were standardized and optimized for the analysis of mixtures prepared from pure standards of DIBOA (1), BOA (2), and MBOA (5). The spectra (UV/vis, NMR, and MS) obtained from the sample were identical to those of the extracts, thus confirming the presence of the allelochemicals generated by the donor plant in the tissue of the target species and verifying the absorption of potentially allelopathic compounds from one species to another in the vicinity.

Once the structures of the compounds had been confirmed as DIBOA (1), BOA (2), and APO (3), their concentrations were determined by HPLC-DAD. An increase in the level of the detected compound in the target plant was observed when the population of donor species was increased. It should be pointed out that the amounts of DIBOA (1), BOA (2), and APO (3) in roots were similar to those in shoots, except for the lowest density, in which DIBOA (1) root levels were higher (Figure 6). Absorption through the root is a process that is governed by several factors, both biotic and abiotic. These factors include concentration in the medium. The metabolic effects caused by the compounds taken up can modify the absorption capacity, which would then vary with time. This phenomenon is related to the capacity for translocation of the chemicals from roots to shoots and the ability to metabolize and detoxify them.

With regard to the molecules to be taken up, their physical properties play a fundamental role in their absorption by roots. The molecular weights and functional groups of these compounds modify their solubilities and are among the determining factors. DIBOA (1), BOA (2), and APO (3) have different solubilities and their different structures also influence the molecular interactions, transport within the plant, and the final access to their molecular target sites of action for phytotoxicity. In the present case, $\operatorname{APO}(3)$ is absorbed the least and DIBOA (1) the most. This fact can be positively correlated with their concentrations in the test environment. However, considering that APO (3) has the lowest solubility in water of the compounds in question, it is not surprising that it has a lower concentration in the target plant.

Phytotoxic Effect on the Target Plant. Once the presence of the allelochemicals in the target plant had been 


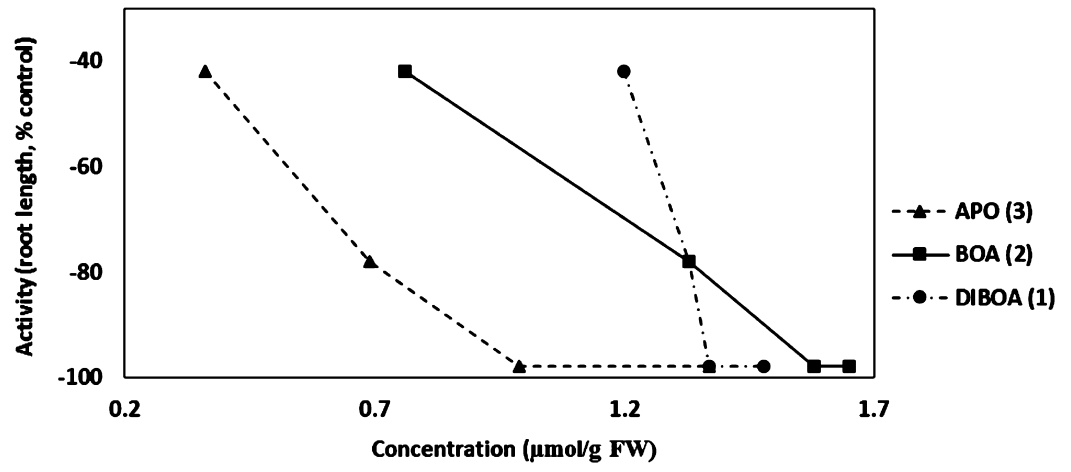

Figure 7. Levels of DIBOA (1), BOA (2), and APO (3) in A. fatua ( $\mu \mathrm{mol} / \mathrm{PFW})$ roots and activity on the root growth parameters.

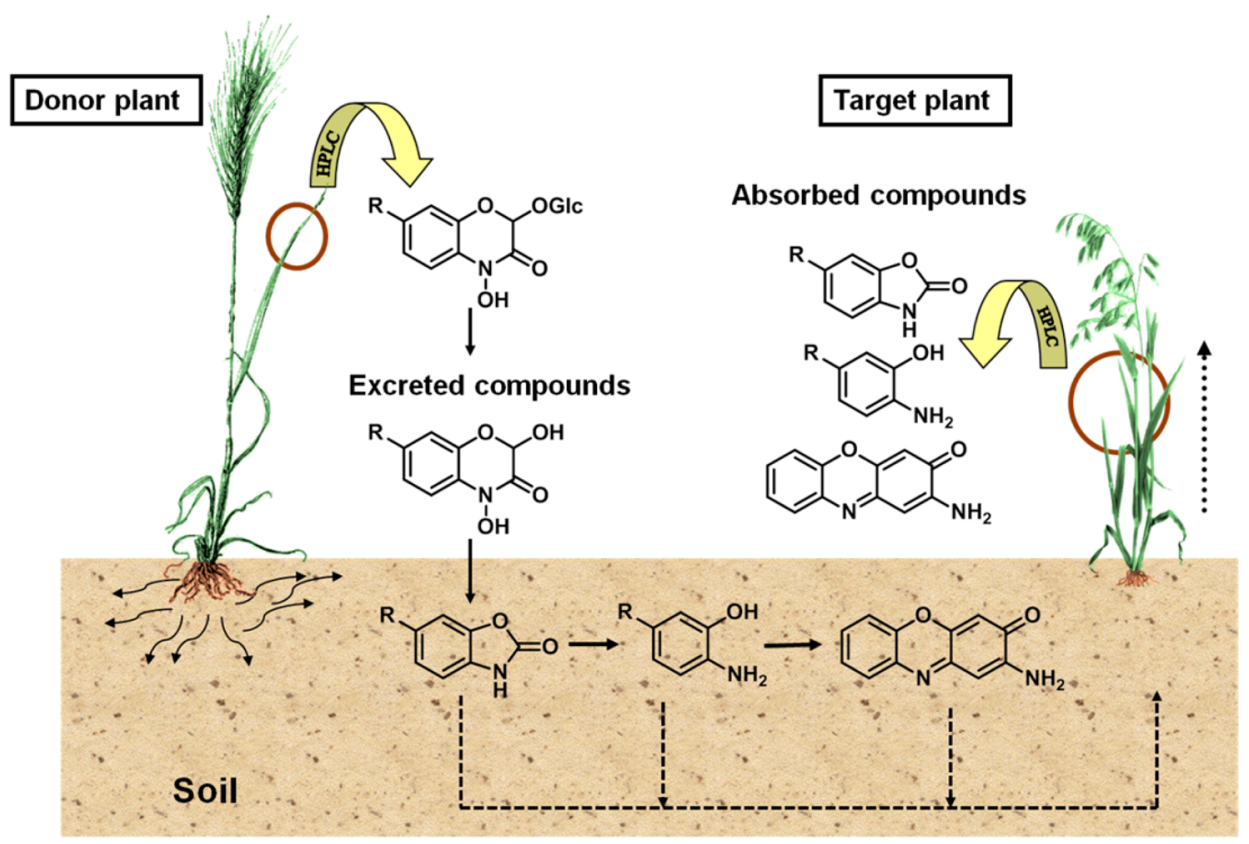

Figure 8. Proposed representation for the allelopathic pathway in the coculture of S. cereale and A. fatua.

confirmed, the dependence on the donor plant density from which they originate had to be described along with their bioactivity. A greater phytotoxic effect was observed as the amounts of allelochemicals found in the target plant tissue increased (Figure 7). The concentration of each chemical inside the plant, along with the resulting phytotoxic effect, followed a typical dose-response relationship. This correlation was observed for the three analyzed compounds DIBOA (1), BOA (2), and APO (3). All of the compounds were found in shoots and roots, albeit at different concentrations. Under these conditions, the higher concentration of DIBOA (1) could indicate that this is the compound that contributes most to the observed effects. Nevertheless, the higher phytotoxicity of APO (3) on A. fatua ${ }^{29}$ implies that this compound would require a lower concentration to achieve the same effect. This result clearly establishes these chemicals as the agents responsible for the phytotoxic effects observed.

In summary, this is the first allelopathy study in which the mediators of the allelopathic phenomenon have been detected in all plant species under investigation and also in the environment in which they were grown (Figure 8). Since this experiment was conducted with living plants and real exudates, the system could capture multicompound interactions that influence the bioavailability of allelochemicals, effects that are otherwise obscured in allelopathy research. ${ }^{38}$

The results provide valuable information for the study of the allelopathic phenomenon and shed light on the role of benzoxazinones and their related chemicals in Gramineae crop-weed interactions. The production, transformation, and degradation processes that occur concomitantly have been fully characterized for the biocommunication process between $S$. cereale and A. fatua. Although the process reported here is a specific example of allelopathy, the results allowed us to show the complexity of this phenomenon, especially in terms of the dynamic nature of exudation, uptake, and degradation processes. The route of the allelochemicals from donor to target plant was fully characterized and the role of benzoxazinones and their degradation products on the interaction was confirmed. We suggest that exudation and uptake dynamics, together with degradation products, are factors that must be considered in studies aimed at the characterization of allelopathic phenomena.

The dynamics of the processes involved in allelopathy are extremely complex and gaining knowledge and understanding requires an in-depth investigation. For example, all experimental variables are time-dependent, from allelochemical structures to concentrations. Allelochemicals should be released 
to the environment and, as a consequence, data on their concentrations within the donor plant are not sufficient to characterize the process. Additionally, degradation products could be more active than the released compound and allelochemicals should also be incorporated into the target plant. This dynamic situation cannot be elucidated by simply recording macroscopic growth effects and analysis of the target plant is also required.

The experimental design described here eliminates the need to consider the soil system as a variable. However, with a view to the possible application of this approach in a field situation, one should consider the role of sorption in the soil and the resulting modification of the bioavailability of components in complex allelochemical mixtures. ${ }^{39}$ Consideration of the experimental results in a field community and at the ecosystem level is crucial in understanding the ecological role of allelochemicals in plant interference. ${ }^{40}$

\section{ASSOCIATED CONTENT}

\section{S Supporting Information}

Chromatographic data of the studies. This material is available free of charge via the Internet at http://pubs.acs.org.

\section{AUTHOR INFORMATION}

\section{Corresponding Author}

*Phone: +34 956.012.770; fax: +34 956.016.193; e-mail: famacias@uca.es.

\section{Funding}

This research was supported by the Ministerio de Economía y competitividad (MINECO) (Project AGL2013-42238-R) and Consejería de Economía Innovación y Ciencia, Junta de Andalucía (Project AGR-5822-2011).

\section{Notes}

The authors declare no competing financial interest.

\section{REFERENCES}

(1) Macias, F. A.; Molinillo, J. M. G.; Varela, R. M.; Galindo, J. C. G. Allelopathy-A natural alternative for weed control. Pest Manage. Sci. 2007, 63, 327-348.

(2) Sinkkonen, A. Ecological Relationships and Allelopathy. In Allelopathy: A Physiological Process with Ecological Implications; Reigosa, M. J., Pedrol, N., González, L., Eds.; Springer: Heidelberg, 2006; pp 373-393.

(3) Bertin, C.; Weston, L. A.; Huang, T.; Jander, G.; Owens, T.; Meinwald, J.; Schroeder, F. C. Grass roots chemistry: meta-tyrosine, an herbicidal nonprotein amino acid. Proc. Natl. Acad. Sci. U.S.A. 2007, 104, 16964-16969.

(4) Wilson, D. S. Biological communitties as funtionally organized units. Ecology 1997, 78, 2018-2024.

(5) Reigosa, M. J.; Sanchez-Moreiras, A.; Gonzalez, L. Ecophysiological approach in allelopathy. Crit. Rev. Plant Sci. 1999, 18, 577-608.

(6) Macías, F. A.; Marin, D.; Oliveros-Bastidas, A.; Simonet, A. M.; Molinillo, J. M. G., Ecological Relevance of the Degradation Processes of Allelochemicals. In Allelopathy: New Concepts \& Methodology; Fujii, Y., Hiradate, S., Eds.; Science Publishers Inc.: Enfield, 2007; pp 91107.

(7) Macias, F. A.; Oliveros-Bastidas, A.; Marin, D.; Castellano, D.; Simonet, A. M.; Molinillo, J. M. G. Degradation studies on benzoxazinoids. Soil degradation dynamics of 2,4-dihydroxy-7methoxy-(2h)-1,4-benzoxazin-3(4h)-one (DIMBOA) and its degradation products, phytotoxic allelochemicals from gramineae. J. Agric. Food Chem. 2004, 52, 6402-6413.

(8) Williamson, G. B.; Obee, E. M.; Weidenhamer, J. D. Inhibition of Schizachyrium scoparium (Poaceae) by the allelochemical hydrocinnamic acid. J. Chem. Ecol. 1992, 18, 2095-2105.
(9) Macias, F. A.; Marin, D.; Oliveros-Bastidas, A.; Molinillo, J. M. G. Rediscovering the bioactivity and ecological role of 1,4-benzoxazinones. Nat. Prod. Rep. 2009, 26, 478-489.

(10) Oliveros-Bastidas, A. d. J.; Macias, F. A.; Marin, D.; CarreraFernandez, C. A.; Molinillo, J. M. G. Decomposition of 2,4-dihydroxy2H-1,4-benzoxazin-3(4H)-one (DIBOA), an allelochemical of grasses. Consequences for measurements of biological activity. Quim. Bras. 2009, 3, 53-60.

(11) Macias, F. A.; Oliveros-Bastidas, A.; Marin, D.; Castellano, D.; Simonet, A. M.; Molinillo, J. M. G. Degradation studies on benzoxazinoids. Soil degradation dynamics of (2R)-2-O- $\beta$-D-glucopyranosyl-4-hydroxy-( $2 H)$ - 1,4-benzoxazin-3(4H)-one (DIBOA-Glc) and its degradation products, phytotoxic allelochemicals from Gramineae. J. Agric. Food Chem. 2005, 53, 554-561.

(12) Adhikari, K. B.; Laursen, B. B.; Gregersen, P. L.; Schnoor, H. J.; Witten, M.; Poulsen, L. K.; Jensen, B. M.; Fomsgaard, I. S. Absorption and metabolic fate of bioactive dietary benzoxazinoids in humans. Mol. Nutr. Food Res. 2013, 57, 1847-1858.

(13) Adhikari, K. B.; Laursen, B. B.; Lærke, H. N.; Fomsgaard, I. S. Bioactive benzoxazinoids in rye bread are absorbed and metabolized in pigs. J. Agric. Food Chem. 2012, 60, 2497-2506.

(14) Burgos, N. R.; Talbert, R. E.; Kim, K. S.; Kuk, Y. I. Growth inhibition and root ultrastructure of cucumber seedlings exposed to allelochemicals from rye (Secale cereale). J. Chem. Ecol. 2004, 30, 671689.

(15) Hussain, M. I.; Reigosa, M. J. Allelochemical stress inhibits growth, leaf water relations, PSII photochemistry, non-photochemical fluorescence quenching, and heat energy dissipation in three C3 perennial species. J. Exp. Bot. 2011, 62, 4533-4545.

(16) Kato-Noguchi, H. Effects of four benzoxazinoids on gibberellininduced $\alpha$-amylase activity in barley seeds. J. Plant Physiol. 2008, 165, 1889-1894.

(17) Woodward, M. D.; Corcuera, L. J.; Helgeson, J. P.; Upper, C. D. Decomposition of 2,4-dihydroxy-7-methoxy-(2H)-1,4-benzoxazin3(4H)-one in aqueous solutions. Plant Physiol. 1978, 61, 796-802.

(18) Fomsgaard, I. S.; Mortensen, A. G.; Carlsen, S. C. K. Microbial transformation products of benzoxazolinone and benzoxazinone allelochemicals: A review. Chemosphere 2004, 54, 1025-1038.

(19) Gagliardo, R. W.; Chilton, W. S. Soil transformation of 2(3H)benzoxazolone of rye into phytotoxic 2-amino-3H-phenoxazin-3-one. J. Chem. Ecol. 1992, 18, 1683-1691.

(20) Kumar, P.; Gagliardo, R.; Chilton, W. Soil transformation of wheat and corn metabolites $\mathrm{MBOA}$ and $\mathrm{DIM}_{2} \mathrm{BOA}$ into aminophenoxazinones. J. Chem. Ecol. 1993, 19, 2453-2461.

(21) Honkanen, E.; Virtanen, A. I. Synthesis of some 1,4-benzoxazine derivatives and their antimicrobial activity. Acta Chem. Scand. 1960, 14, 1214-1217.

(22) Escobar, C.; Sicker, D.; Niemeyer, H. Evaluation of dimboa analogs as antifeedants and antibiotics towards the aphid Sitobion avenae in artificial diets. J. Chem. Ecol. 1999, 25, 1543-1554.

(23) Schulz, M.; Friebe, A.; Kueck, P.; Seipel, M.; Schnabl, H. Allelopathic effects of living quack grass (Agropyron repens L.). Identification of inhibitory allelochemicals exuded from rhizome borne roots. Angew. Bot. 1994, 68, 195-200.

(24) Wolf, R. B.; Spencer, G. F.; Plattner, R. D. Benzoxazolinone, 2,4 dihydroxy-1,4-benzoxazin-3-one, and its glucoside from Acanthus mollis seeds inhibit velvetleaf germination and growth. J. Nat. Prod. $1985,48,59-63$.

(25) Bravo, H. R.; Lazo, W. Antialgal and antifungal activity of natural hydroxamic acids and related compounds. J. Agric. Food Chem. 1996, 44, 1569-1571.

(26) Sahi, S. V.; Chilton, M. D.; Chilton, W. S. Corn metabolites affect growth and virulence of Agrobacterium tumefaciens. Proc. Natl. Acad. Sci. U.S.A. 1990, 87, 3879-3883.

(27) Macias, F. A.; Marin, D.; Oliveros-Bastidas, A.; Castellano, D.; Simonet, A. M.; Molinillo, J. M. G. Structure-activity relationships (SAR) studies of benzoxazinones, their degradation products and analogues. phytotoxicity on standard target species (STS). J. Agric. Food Chem. 2005, 53, 538-548. 
(28) Eljarrat, E.; Guillamon, M.; Seuma, J.; Mogensen, B. B.; Fomsgaard, I. S.; Olivero-Bastidas, A.; Macias, F. A.; Stochmal, A.; Oleszek, W.; Shakaliene, O.; Barcelo, D. First European interlaboratory study of the analysis of benzoxazinone derivatives in plants by liquid chromatography. J. Chromatogr. A 2004, 1047, 69-76.

(29) Macias, F. A.; Marin, D.; Oliveros-Bastidas, A.; Chinchilla, D.; Simonet, A. M.; Molinillo, J. M. G. Isolation and synthesis of allelochemicals from gramineae: benzoxazinones and related compounds. J. Agric. Food Chem. 2006, 54, 991-1000.

(30) Schulz, M.; Marocco, A.; Tabaglio, V.; Macias, F. A.; Molinillo, J. M. G. Benzoxazinoids in rye allelopathy-From discovery to application in sustainable weed control and organic farming. J. Chem. Ecol. 2013, 39, 154-174.

(31) Mathiassen, S. K.; Kudsk, P.; Mogensen, B. B. Herbicidal effects of soil-incorporated wheat. J. Agric. Food Chem. 2006, 54, 1058-1063. (32) Olivero-Bastidas, A. Allelopathic studies from Gramineae. Benzoxazinones as allelochemicals. $\mathrm{PhD}$ dissertation, University of Cádiz, Spain, 2006.

(33) Wu, H.; Haig, T.; Pratley, J.; Lemerle, D.; An, M. Allelochemicals in wheat (Triticum aestivum L.): production and exudation of 2,4-dihydroxy-7-methoxy-1,4-benzoxazin-3-one. J. Chem. Ecol. 2001, 27, 1691-1700.

(34) Macias, F. A.; Chinchilla, N.; Varela, R. M.; Oliveros-Bastidas, A.; Marin, D.; Molinillo, J. M. G. Structure-activity relationship studies of benzoxazinones and related compounds. Phytotoxicity on Echinochloa crus-galli (L.) P. Beauv. J. Agric. Food Chem. 2005, 53, 4373-4380.

(35) Bais, H. P.; Walker, T. S.; Stermitz, F. R.; Hufbauer, R. A.; Vivanco, J. M. Enantiomeric-dependent phytotoxic and antimicrobial activity of $( \pm)$-catechin. A rhizosecreted racemic mixture from spotted knapweed. Plant Physiol. 2002, 128, 1173-1179.

(36) Kobayashi, K. Factors affecting phytotoxic activity of allelochemicals in soil. Weed Biol. Manage. 2004, 4, 1-7.

(37) An, M.; Liu, D. L.; Johnson, I. R.; Lovett, J. V. Mathematical modelling of allelopathy: II. The dynamics of allelochemicals from living plants in the environment. Ecol. Model. 2003, 161, 53-66.

(38) Tharayil, N.; Bhowmik, P. C.; Xing, B. Bioavailability of allelochemicals as affected by companion compounds in soil matrices. J. Agric. Food Chem. 2008, 56, 3706-3713.

(39) Tharayil, N.; Bhowmik, P. C.; Xing, B. Preferential sorption of phenolic phytotoxins to soil: implications for altering the availability of allelochemicals. J. Agric. Food Chem. 2006, 54, 3033-3040.

(40) Inderjit; Callaway, R. M. Experimental designs for the study of allelopathy. Plant Soil 2003, 256, 1-11. 